UNIVERSIDADE DE SÃO PAULO

INSTITUTO DE PSICOLOGIA

WALTER LISBOA OLIVEIRA

Produção de Sentido na prática profissional de Auxiliares

e Técnicos de Enfermagem 


\title{
Produção de Sentido na prática profissional de Auxiliares e Técnicos de Enfermagem
} (Versão Corrigida)

\author{
Tese apresentada ao Instituto de \\ Psicologia da Universidade de São Paulo \\ como parte dos requisitos para obtenção \\ do título de Doutor em Psicologia. \\ Área de Concentração: \\ Psicologia Clínica. \\ Orientador: \\ Prof. Dr. Avelino Luiz Rodrigues.
}


Autorizo a reprodução e divulgação parcial deste trabalho, por qualquer meio convencional ou eletrônico, para fins de estudo e pesquisa, desde que citada a fonte.

Catalogação na publicação

Biblioteca Dante Moreira Leite

Instituto de Psicologia da Universidade de São Paulo

Oliveira, Walter Lisboa.

Produção de sentido na prática profissional de auxiliares e técnicos de enfermagem / Walter Lisboa Oliveira; orientador Avelino Luiz Rodrigues. -- São Paulo, 2015.

$164 \mathrm{f}$.

Tese (Doutorado - Programa de Pós-Graduação em Psicologia. Área de Concentração: Psicologia Clínica) - Instituto de Psicologia da Universidade de São Paulo.

1. Sentido do trabalho 2. Síndrome de Burnout 3. Medicina psicossomática 4. Auxiliares e técnicos de enfermagem 5. Qualidade de vida no trabalho I. Título.

HD4831 


\section{FOLHA DE APROVAÇÃO}

Nome: Oliveira, Walter Lisboa

Título: Produção de Sentido na prática profissional de Auxiliares e Técnicos de Enfermagem

Tese apresentada ao Instituto de Psicologia da Universidade de São Paulo para obtenção do título de Doutor em Psicologia Clínica.

Aprovado em

\section{Banca Examinadora}

Prof. Dr.

Instituição Assinatura

Prof. Dr.

Instituição Assinatura

Prof. Dr.

Instituição Assinatura

Prof. Dr.

Instituição Assinatura

Prof. Dr.

Instituição Assinatura 
DEDICATÓRIA

A meus pais, Luiz Hermínio e Maria de Fátima, meus irmãos Luiz Alves e Vitor Lisboa e a meu grande amor Iris De Vita, pessoas especiais que acompanham minha caminhada, sempre apoiando e sendo constantes fontes de alegria. 


\section{AGRADECIMENTOS}

A meus pais Luiz Hermínio de Aguiar Oliveira e Maria de Fátima Lisboa Oliveira, pelo inestimável amor, apoio e carinho durante toda minha vida.

A meus irmãos Luiz Alves e Vitor Lisboa, que apesar da distância, sempre se fizeram presentes ajudando-me tanto neste trabalho, quanto em outras situações da minha vida.

A meu grande amor Iris De Vita, por encher meus dias de alegrias, pela importante ajuda ao longo dessa caminhada e por estar sempre presente em todos os momentos, apoiando, acreditando e compartilhando sonhos.

A todos meus amigos, tios, primos e familiares pelo apoio e carinho manifestados ao longo dessa jornada, em especial aos meus avós Luiz Alves (in memorian) e Maria Hermínia (in memorian), Walter Lisboa (in memorian) e Helena Maria Franco (in memorian), minha tia avó Beltiz Franco (in memorian).

À Tânia Lúcia Scavone, Wolmer Brito e Soraya Vila, por terem me acolhido de maneira carinhosa, sendo importantes figuras de apoio e conforto familiar, imprescindíveis aos meus dias em São Paulo.

A meu orientador, Prof. Dr. Avelino Luiz Rodrigues, meus sinceros agradecimentos pelas preciosas orientações, incentivo à minha formação profissional e decisivo apoio tanto do ponto de vista acadêmico quanto pessoal nesta caminhada.

À Profa. Dra. Elisa Maria Parahyba Campos pelo acolhimento carinhoso e estímulo ao desenvolvimento deste trabalho.

À Prof ${ }^{a}$ Dra. Cristina Limongi-França, por todo apoio, generosidade e valiosos conselhos ao longo de todo doutorado.

Ao Prof. Dr. Sigmar Malvezzi, pela disponibilidade, sábios ensinamentos e constantes apoio e incentivo.

À Profa. Dra Ivete de Souza Yavo pelos importantes conselhos e auxílio nos primeiros passos da pesquisa.

À psicóloga Mia Olsen de Almeida por todo apoio imprescindível nesta caminhada.

Aos meus amigos dos laboratórios Chronos-USP e SuCor que contribuíram de forma para o êxito deste trabalho. 
Aos meus professores do Curso de Psicologia da Universidade Federal de Sergipe, em especial meus orientadores ao longo da minha vida acadêmica, grandes incentivadores e responsáveis pelos primeiros passos deste caminho que venho percorrendo: Célia Vieira, Cybele Ramalho, Daniel Coelho, Marcus Eugênio, Maria Benedita, Ricardo Barreto e Sheila Barboza.

À Universidade de São Paulo, em especial ao Instituto de Psicologia e ao Hospital Universitário, pelas condições oferecidas para realização deste trabalho.

Aos profissionais do Hospital Universitário da Universidade de São Paulo, em especial à Profa. Dra. Heloisa Helena Ciqueto Peres, Diretora do Departamento de Enfermagem pelo imprescindível apoio ao longo da jornada.

Aos chefes de Enfermagem das unidades hospitalares em que atendi pacientes e familiares, Cleide Maria Caetano Baptista, da Clínica Cirúrgica, e Paulo Carlos Garcia, da Unidade de Terapia Intensiva, e toda equipe, pelo importante e valoroso apoio aos atendimentos e à pesquisa de Mestrado e Doutorado.

Aos colegas do hospital, o Enfermeiro Fábio José da Silva e às secretárias do Departamento Médico Maria Akie Kowara e Cristiana Oliveira Gomes, pelos frequentes esclarecimentos, apoio e incentivo à pesquisa.

Ao Sr. Pedro Nepomuceno do Carmo, Diretor de Pessoal do Hospital Universitário, pela generosa disponibilidade e prontidão, auxiliando esta pesquisa.

Aos funcionários da USP, da Comissão de Pós-Graduação, Secretaria de Psicologia Clínica e Biblioteca, pela paciência e disponibilidade em ajudar, em especial a Cláudia Rocha pelos atenciosos esclarecimentos.

Às secretárias do Comitê de Ética do Instituto de Psicologia, Silvia Aparecida Sochiarelli, e do Hospital Universitário, Wilma Monteiro Frésca, pelas cuidadosas e pacientes orientações à pesquisa.

A Luiz Silva dos Santos, do setor de Métodos Quantitativos do IP-USP, pela paciência com meu aprendizado, e pelo atencioso cuidado com os dados desta pesquisa.

À CAPES pelo apoio financeiro fundamental para a realização deste curso de pósgraduação.

A todos os participantes da pesquisa, essenciais ao seu desenvolvimento, por compartilharem suas histórias e experiências de vida, revelando aspectos íntimos de sua existência, acreditando e dando significado ao nosso trabalho.

A todos que contribuíram direta e indiretamente para a conclusão desta importante etapa da minha vida. 


\section{RESUMO}

Oliveira, W. L. (2015). Produção de sentido na prática profissional de auxiliares e técnicos de enfermagem (Tese de doutorado). Universidade de São Paulo.

O objetivo da pesquisa foi compreender como os auxiliares e técnicos produzem sentidos sobre seu trabalho e sua vida. Entendendo que experiências de sofrimento emocional e transtornos psicossociais podem intervir na produção de sentido, este trabalho investigou também a qualidade de vida no trabalho e a existência de burnout. Foi utilizado o método quantitativo e qualitativo. Os instrumentos utilizados foram o Inventário de Burnout de Maslach para profissionais de saúde (MBI-HSS), a escala BPSO-96 e um protocolo de entrevista semidirigida. A pesquisa foi realizada no Hospital Universitário da Universidade de São Paulo, as escalas foram aplicadas a 166 profissionais de saúde, dentre técnicos, auxiliares e outros profissionais do hospital e foram entrevistados 20 destes participantes. As escalas foram analisadas estatisticamente tanto na amostra inteira quanto segmentada em dois grupos, um de auxiliares e técnicos de enfermagem e um grupo de outros profissionais para verificar se os fenômenos observados no primeiro grupo são específicos dessa categoria profissional. As entrevistas foram categorizadas e analisadas. Os resultados apontaram alto nível de despersonalização, médio de exaustão, baixa realização pessoal.e nível médio de satisfação com a Qualidade de Vida no Trabalho, não havendo diferença estatística significativa entre os dois grupos. O momento de crise política vivenciado pelo hospital durante a coleta de dados pode ter interferido nos resultados, uma vez que as entrevistas semidirigidas revelaram insegurança, luto pela perda do hospital idealizado e insegurança quanto ao seu futuro profissional. Quanto aos sentidos produzidos, os profissionais entendem que sua prática é imprescindível para o tratamento médico, percebem-se como elo essencial entre paciente e equipe multidisciplinar e referem reconhecimento social pela atuação na área da saúde. Os auxiliares e técnicos de enfermagem revelaram ainda satisfação pelo ato de cuidar, tendo na recuperação dos pacientes e seu reconhecimento e gratidão, importante fonte de produção de sentido da prática profissional.

Palavras-Chave: sentido do trabalho, síndrome de burnout, medicina psicossomática, auxiliares e técnicos de enfermagem, qualidade de vida no trabalho. 


\begin{abstract}
Oliveira, W. L. (2015). The meaning of work in professional practice of nursing assistants. (Doctorate Thesis). Universidade de São Paulo.

The objective of the research was comprehending how they give meaning to their work and life. Seeing that experiences of emotional suffering and psychosocial disorders may interfere in the meaning of work, this research has also looked into the quality of work life and the existence of burnout syndrome. Quantitative and qualitative methods were used. The research instruments applied were Maslach Burnout Inventory- Human Services Survey (MBI-HSS), the BPSO-96 scale and a guide of semi structured interviews. The research was carried out at the University Hospital in São Paulo, the scales were applied to 166 health professionals, among assistants and other health professionals. 20 participants were interviewed. The scales were statically analyzed on the whole and segmented samples in two groups, one with nursing assistants and other one with other professionals, so both results were compared. The interviews were categorized and analyzed. The results have shown a high level of depersonalization, a medium level of exhaustion, a low level of personal fulfillment and a medium level of satisfaction with the quality of work life, there has not been a representative statistical difference between the two groups. The hospital was going through a political crisis during the data collection which may have interfered in the results, once the semi structured interviews have revealed insecurity, mourning over the loss of the idealized hospital and feeling of uncertainty towards their professional future. With regard to the meaning of work, the professionals understand that their practice is indispensable for medical treatments. They see themselves as a necessary link between patients and multidisciplinary teams. They also mention social recognition for the work in the health area and satisfaction when taking care of patients, seeing their recovery and gratitude as an important source of meaning in the professional practice.
\end{abstract}

Key Words: meaning of work, burnout syndrome, psychosomatic medicine, nursing assistants, quality of work life 


\section{LISTA DE ILUSTRAÇÕES}

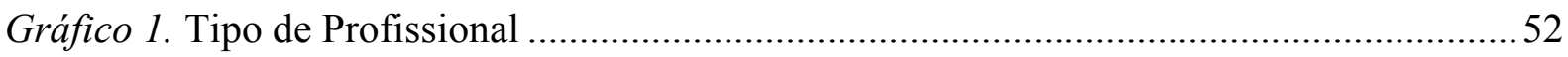

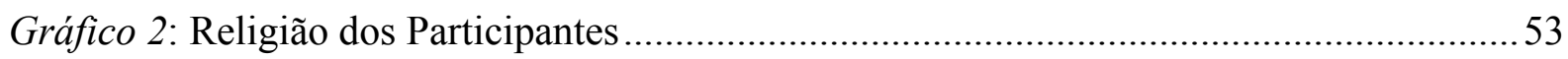

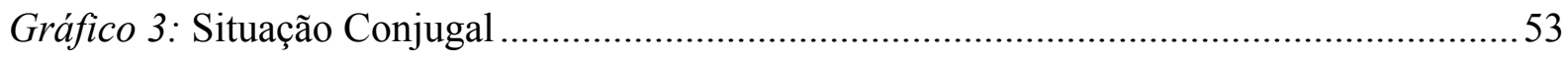

Gráfico 4. Distribuição percentual nas Unidades Hospitalares ............................................... 55

Gráfico 5. Distribuição Percentual por Turno de Trabalho...................................................... 56

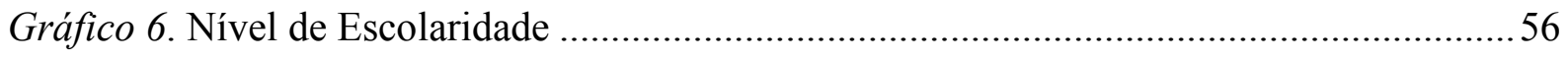

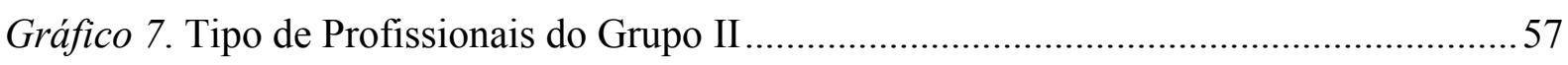

Gráfico 8. Jornada de Trabalho dos outros Profissionais de Saúde ........................................58

Gráfico 9. Escolaridade do Grupo I (Auxiliares e Técnicos) ....................................................5

Gráfico 10. Escolaridade do Grupo II (Outros Profissionais de Saúde) .................................59

Gráfico 11. Cursos de Ensino Superior dos Profissionais do Grupo I .....................................60

Gráfico 12. Cursos de Ensino Superior dos Profissionais do Grupo II .................................... 60

Gráfico 13. Níveis de exaustão emocional entre auxiliares e técnicos de enfermagem.......... 104

Gráfico 14. Níveis de exaustão emocional entre os outros profissionais de saúde .................. 104

Gráfico 15. Níveis de despersonalização entre os auxiliares e técnicos de enfermagem....... 105

Gráfico 16. Níveis de despersonalização entre os outros profissionais de saúde..................... 106

Gráfico 17. Níveis de realização pessoal entre os auxiliares e técnicos de enfermagem........ 107

Gráfico 18. Níveis de realização pessoal entre os outros profissionais de saúde.................... 107

Gráfico 19: Médias dos Escores referentes aos índices de satisfação de QV ........................ 108

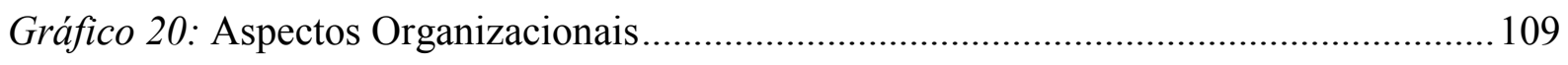

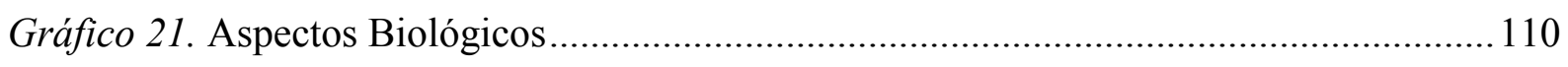

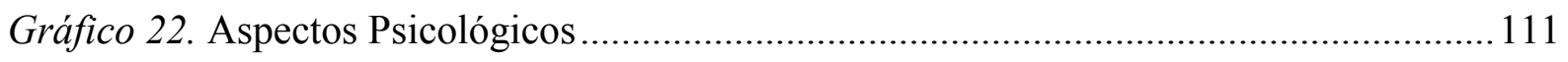

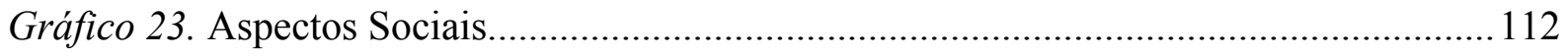

Gráfico 24. Estado Pessoal de Qualidade de Vida no Trabalho............................................ 113

Gráfico 25. Percepção de Melhorias na Qualidade de Vida no Trabalho entre Auxiliares e

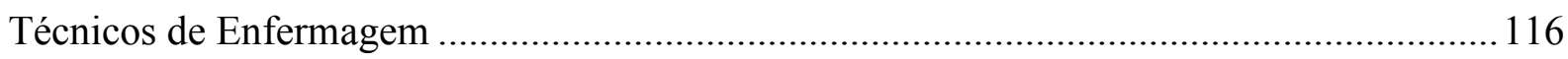

Gráfico 26. Percepção de Melhorias na Qualidade de Vida no Trabalho entre outros .......... 117 Gráfico 27. Melhorias em qualidade de vida no trabalho citadas pelos auxiliares e técnicos de

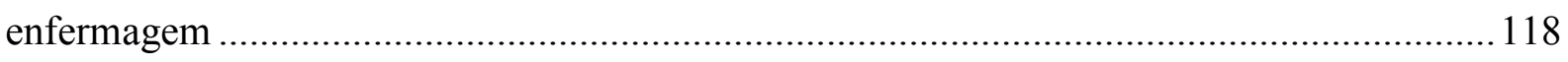

Gráfico 28. Melhorias em qualidade de vida no trabalho citadas pelos auxiliares e técnicos de enfermagem 


\section{LISTA DE TABELAS}

Tabela 1. Padrão de pontuação para diagnóstico das dimensões da síndrome de burnout pelo

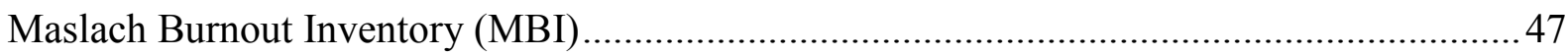

Tabela 2. Idade: Análise Descritiva na Amostra Completa...................................................52

Tabela 3. Tempo em anos de trabalho com a função profissional ..........................................54

Tabela 4. Tempo em anos de Trabalho na Instituição ............................................................. 54

Tabela 5. Tempo em anos de Atuação na área da saúde ........................................................ 54

Tabela 6. Padrão de pontuação para avaliação das dimensões da síndrome de burnout pelo

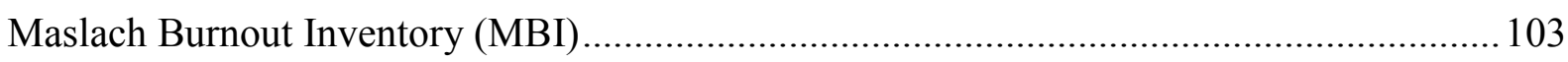

Tabela 7. Exaustão Emocional - Análise descritiva da amostra ............................................. 103

Tabela 8. Exaustão Emocional - Análise descritiva da amostra segmentada ........................ 103

Tabela 9. Despersonalização - Análise Descritiva da Amostra Inteira.................................. 104

Tabela 10. Despersonalização - Análise Descritiva da Amostra Segmentada........................ 105

Tabela 11. Realização Pessoal - Análise Descritiva da Amostra Inteira ............................... 106

Tabela 12. Realização Pessoal - Análise da Amostra Segmentada ...................................... 106

Tabela 13. Três palavras que expressam Qualidade de Vida no Trabalho ............................ 114

Tabela 14. Total de Ocorrências em Doença......................................................................... 114

Tabela 15. Ocorrências de Problemas de Saúde................................................................... 115

Tabela 16. Sintomas ou Manifestações de problemas de saúde ............................................ 115

Tabela 15. Comparação dos escores do MBI-HSS com estudo de Yavo (2012) .................... 123

Tabela 16. Comparativo da distribuição dos níveis de burnout com estudo de Yavo (2012) 124

Tabela 17. Comparativo dos escores do MBI-HSS com estudo de Moreira et al (2009) ...... 124

Tabela 18. Comparativo da distribuição dos níveis de burnout com estudo de Moreira et al (2009) 


\section{LISTA DE SIGLAS}

BPSO-96 Indicadores empresariais de Qualidade de Vida no Trabalho (LimongiFrança,1996)

BPSO-H Indicadores biopsicossociais de Qualidade de Vida no Trabalho em Hospital

CIPA Comissão Interna de Prevenção de Acidentes

COFEN Conselho Federal de Enfermagem

CONEP Comissão Nacional de Ética em Pesquisa

HU-USP Hospital Universitário da Universidade de São Paulo

IP-USP Instituto de Psicologia da Universidade de São Paulo

MBI-HSS Maslach Burnout Inventory - Human Service Survey

QVT Qualidade de Vida no Trabalho

SIPAT Sistemas de Prevenção de Acidente do Trabalho

USP Universidade de São Paulo

UTI Unidade de Terapia Intensiva 


\section{SUMÁRIO}

\section{PALAVRAS INICIAIS}

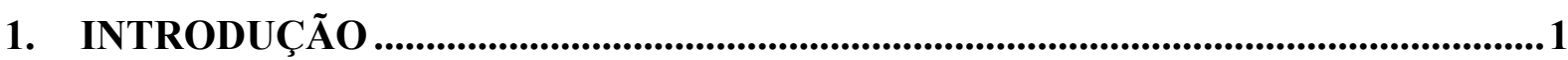

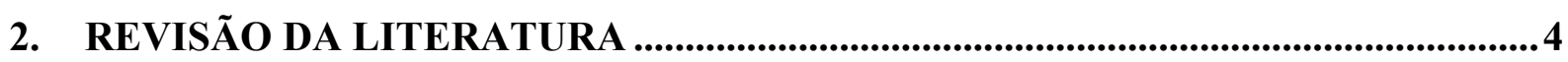

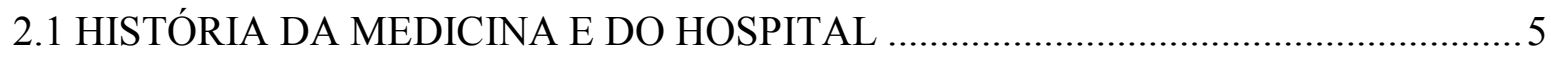

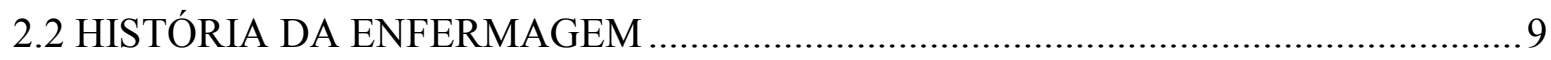

2.3 - HISTÓRIA DA MEDICINA E DA ENFERMAGEM NO BRASIL.............................. 11

2.4 HISTÓRIA DOS AUXILIARES E TÉCNICOS DE ENFERMAGEM ………...............16

2.5 PRODUÇÃO DE SENTIDOS E SENTIDO DO TRABALHO......................................19

2.6 O ESTRESSE E A SÍNDROME DE BURNOUT ………............................................

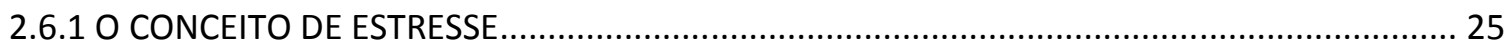

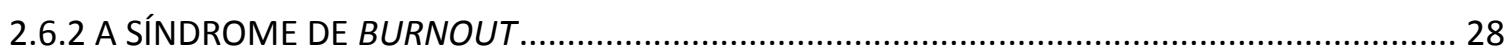

2.7 O HOSPITAL E A EQUIPE DE ENFERMAGEM ……………….............................. 35

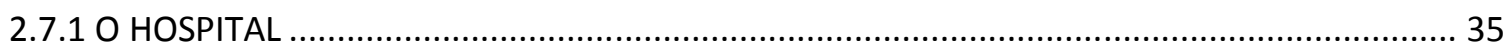

2.7.3 ENFERMEIROS, AUXILIARES E TÉCNICOS .................................................................... 36

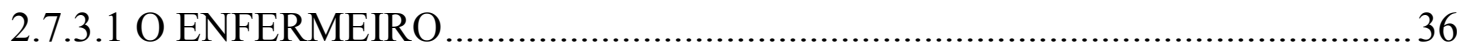

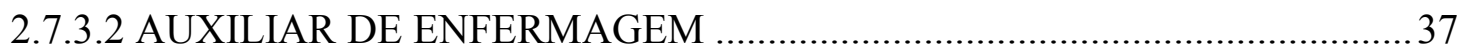

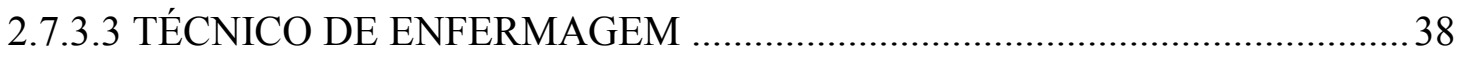

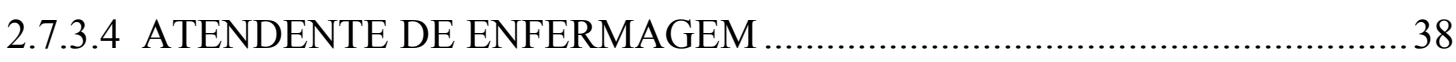

3. JUSTIFICATIVA ........................................................................................................... 40

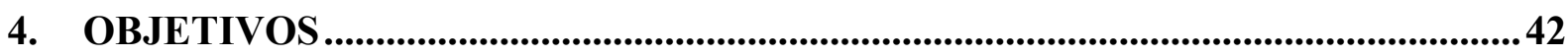

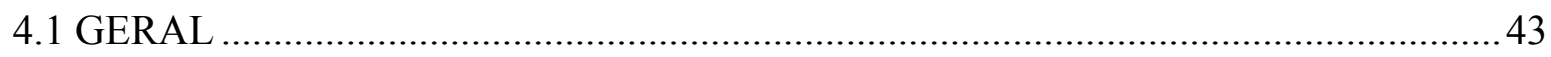

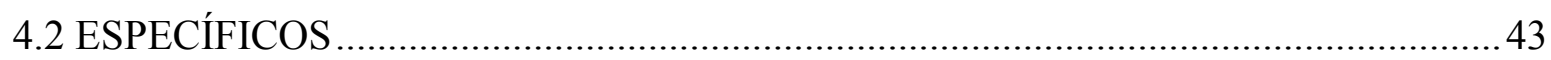

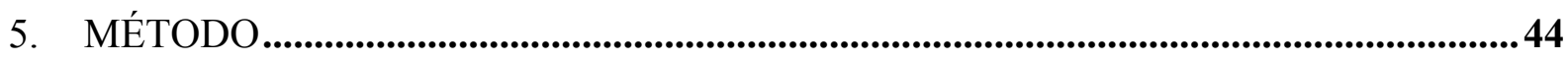

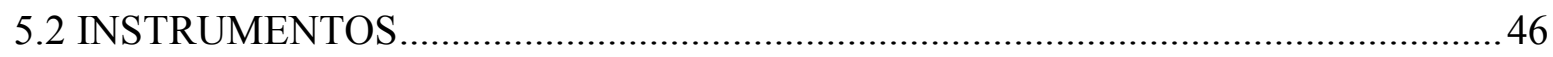

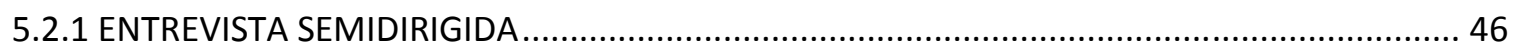

5.2.2 MASLACH BURNOUT INVENTORY - HUMAN SURVEY SERVICE (MBI-HSS) ......................... 47

5.2.3 ESCALA DE AVALIAÇÃO DE QUALIDADE DE VIDA NO TRABALHO EM HOSPITAL - BPSOH... 48

5.3 LOCAL DA PESQUISA: O HOSPITAL UNIVERSITÁRIO ........................................49

5.4.1 SEGMENTAÇÃO DA AMOSTRA ................................................................................... 57

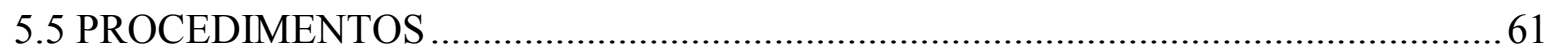




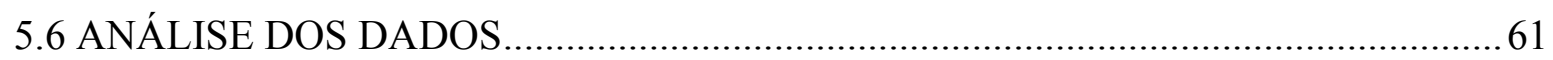

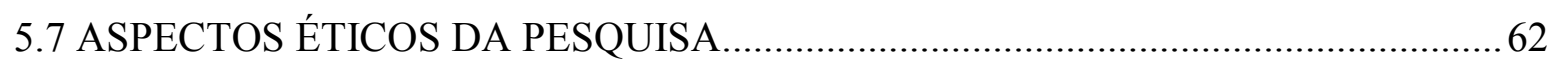

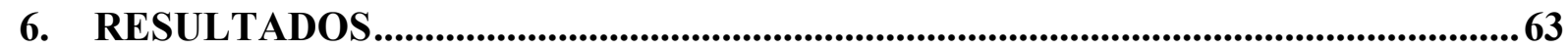

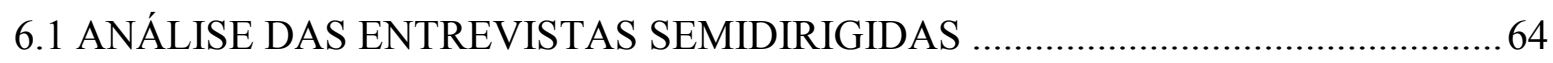

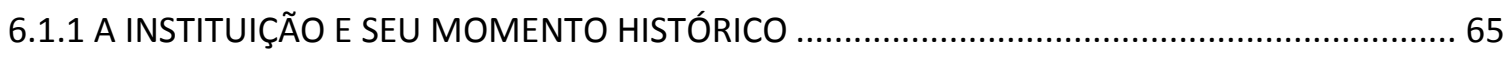

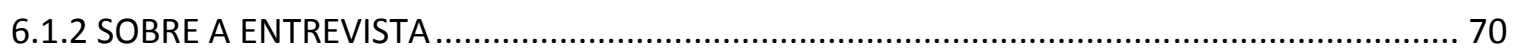

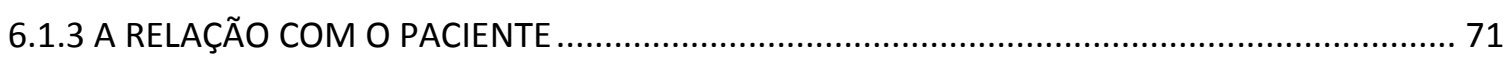

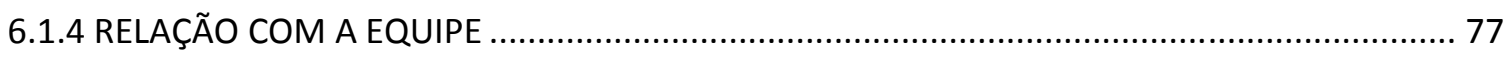

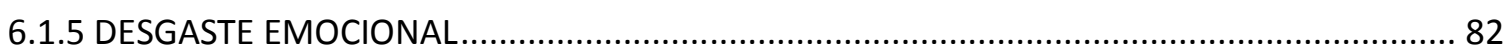

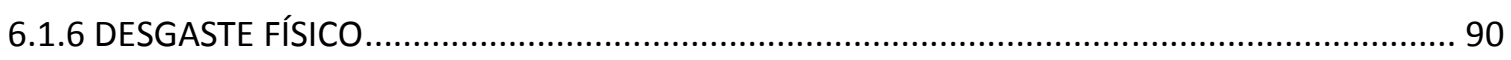

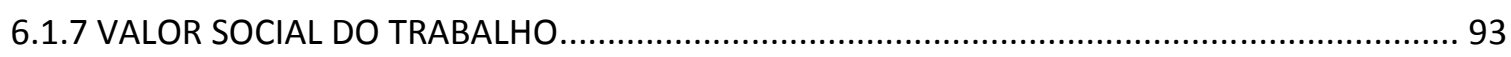

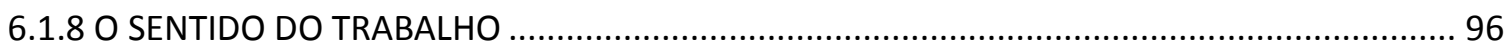

6.2 ANÁLISE DOS DADOS OBTIDOS ATRAVÉS DA MASLACH BURNOUT INVENTORY - HUMAN SERVICES SURVEY (MBI-HSS) ...................................... 102

6.3 ESCALA DE AVALIAÇÃO DE QUALIDADE DE VIDA NO TRABALHO BPSO-H 107

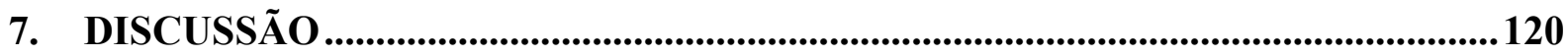

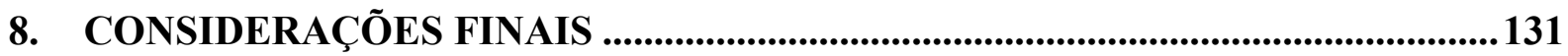

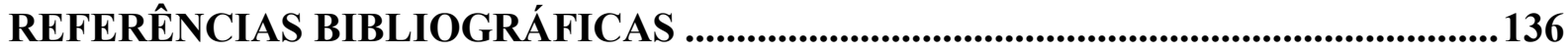

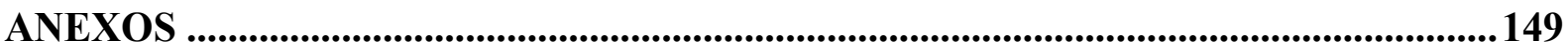

ANEXO A - Protocolo de Entrevista Semidirigida - Produção de Sentidos na Prática Profissional de Auxiliares e Técnicos de Enfermagem ................................................ 150

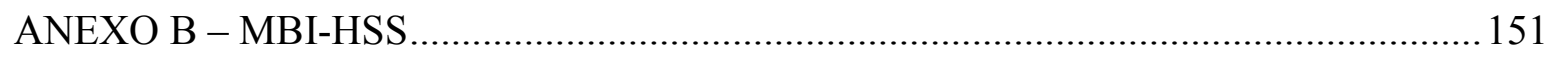

ANEXO C - BPSOH - Indicadores biopsicossociais de Qualidade de Vida no Trabalho em

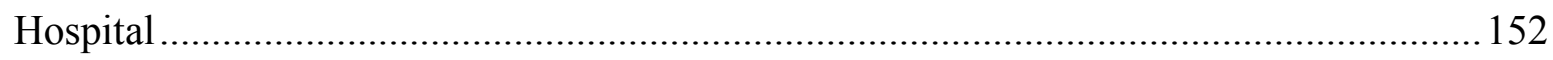

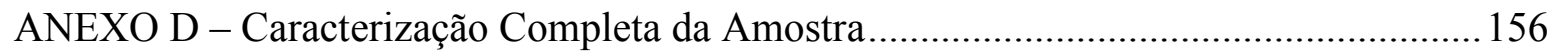

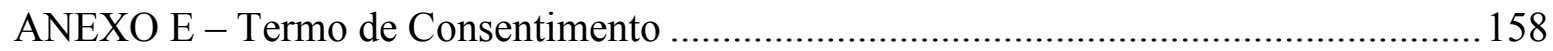

Anexo G - Médias dos escores das Questões do MBI ........................................................... 162

ANEXO H- Médias dos Escores de Satisfação no BPSOH.............................................. 163 